\title{
Des armes, du sport, des hommes... et des femmes
}

Genre et techniques dans le tir sportif en Suisse

Weapons, sports, men... and women. Gender and techniques in shooting sports in Switzerland

\section{Solène Froidevaux}

\section{(2) OpenEdition}

1 Journals

\section{Édition électronique}

URL : http://journals.openedition.org/artefact/3246

DOI : 10.4000/artefact.3246

ISSN : 2606-9245

Éditeur:

Association Artefact. Techniques histoire et sciences humaines, Presses universitaires du Midi

\section{Édition imprimée}

Date de publication : 15 mars 2019

Pagination : 195-214

ISBN : 978-2-8107-0623-5

ISSN : 2273-0753

\section{Référence électronique}

Solène Froidevaux, «Des armes, du sport, des hommes... et des femmes », Artefact [En ligne], 9 | 2018, mis en ligne le 04 mars 2020, consulté le 27 novembre 2020. URL : http://journals.openedition.org/ artefact/3246 ; DOI : https://doi.org/10.4000/artefact.3246

\section{(ब) $\odot \Theta$}

Artefact, Techniques, histoire et sciences humaines est mise à disposition selon les termes de la Licence Creative Commons Attribution - Pas d'Utilisation Commerciale - Pas de Modification 4.0 International. 


\section{Des armes, du sport, des hommes... et des femmes}

Genre et techniques dans le tir sportif en Suisse

\section{Solène Froidevaux}

\section{Résumé}

La pratique sportive du tir avec armes à feu et à l'arc, majoritairement investie par les hommes, demande des techniques et des postures corporelles socialement associées à la masculinité : prendre de l'espace, avoir de l'assurance dans ces gestes, et surtout manipuler des armes. Cet article s'intéresse, à partir d'une recherche ethnographique, à la manière dont les femmes et les hommes s'intègrent dans ce milieu sportif et en quoi la matérialité environnante marque leur expérience corporelle et l'acquisition de techniques particulières. Si une répartition genrée des armes est visible, basée sur des croyances différenciées de ce qu'un corps d'homme et de femme est capable de faire, l'étude du tir montre également que des éléments contextuels peuvent contribuer à déstabiliser les représentations sociales genrées et permettre aux femmes de manipuler des objets et des techniques, qui leur étaient rendus socialement inaccessibles.

\section{Mots-clés}

armes, corps, genre, manipulation, technique, tir sportif

99 Solène Froidevaux, « Des armes, du sport, des hommes... et des femmes. Genre et techniques dans le tir sportif en Suisse », Artefact, 9, 2018, p. 195-214. 


\section{Weapons, sports, men... and women. Gender and techniques in shooting sports in Switzerland}

\section{Abstract}

Shooting sports are mostly practiced by men in Switzerland. These sportive practices imply techniques and bodily postures that are socially linked to masculinity: take place, be bodily-confident and above all handle weapons. From an ethnographic inquiry, this article tackles the way that women and men are bodily integrated to this space and how the materiality plays a role in the experience of learning and practicing. A sexual division of weapons is visible on the fieldwork, based on differential beliefs about the capability of women's bodies and men's bodies. However, the contextual elements can contribute to challenge gendered social representations and enable women to handle objects and techniques that were socially inaccessible, the weapons.

\section{Keywords}

bodies, gender, handling, shooting sport, technique, weapons 
\ک C'est sous un soleil de plomb que j'arrive au terrain de foot, réservé le temps des entraînements hebdomadaires du club à la pratique du tir à l'arc. Maurice est ravi d'être celui qui va me faire toucher un arc pour la première fois. C'est en effet ma séance d'initiation et je suis moi-même excitée. Maurice me tend très vite un arc d'initiation et me dit qu'il ne va pas trop parler car « ce que je veux aujourd'hui, ce ne sont pas des résultats mais que tu sentes ton arc, que tu sentes ce que tu fais ». Comme il le souligne, " il s'agit avant tout d'avoir une posture en T et stable». Perpendiculaire à la cible et à quelques mètres de celle-ci, je me retrouve donc yeux fermés à devoir trouver ma " position naturelle ", celle avec laquelle je ne bouge pas même si on me bouscule. Il me dit également d'avoir " la rage ", d' " avoir l'intention de me battre avec la cible » : "Quand tu tires à l'arc, tu dois être fière [il mime une musculature imposante en gonflant le torse], tu dois allonger ton haut du corps au maximum, prendre de la place.

et extrait est issu de mon terrain ethnographique, que j'ai effectué
durant deux ans et demi en Suisse dans le cadre de ma thèse, sur
le tir à l'arc et le tir avec armes à feu en Suisse ${ }^{1}$. Le tir à l'arc et le
tir avec armes à feu demandent, comme toute pratique, un apprentissage.
Ils supposent également un espace et celui-ci est intéressant à questionner
du point de vue du genre et de la mise en mouvement des corps faisant
du tir. En effet, comme le montre l'extrait ci-dessus, la pratique du tir
implique une manière de tenir et de mouvoir son corps, et d'incarner des
"qualités " telles que l'agressivité et la fierté, qui sont associées socialement

\footnotetext{
1. Dans le cadre de ma recherche doctorale, j'ai mené vingt-six entretiens sous forme de récit de vie (50\% femmes, $50 \%$ hommes; douze pratiquant le tir à l'arc, douze le tir avec armes à feu et deux personnes pratiquant les deux ; moyenne d'âge 45 ans, représentative de la population des tireur-se-s observée). J'ai observé et participé à plusieurs scènes de pratiques de tir à l'arc et avec armes à feu dans plusieurs clubs et événements (entraînements dans des clubs de village et de ville ; compétitions régionales, nationales et internationales sur sol suisse ; fêtes de tir ; événements autour du tir comme par exemple la bourse annuelle des armes ; appartements de collectionneurs), ce qui représente $120 \mathrm{~h}$ d'observation. En Suisse, le tir à l'arc est organisé au niveau national par l'Association suisse de Tir à l'Arc, qui se définit légalement comme une fédération organisée sous la forme d'une association (voir Statuts sur le site www.swissarchery.ch) et ne fait pas partie de la Fédération suisse de Tir, qui ne concerne que le tir avec armes à feu. Cependant, les cours de moniteurs/trices de tir pour les jeunes (Jeunesse et Sport) de la Fédération sont regroupés et les moniteurs/trices se familiarisent donc avec tous les types de tir.
} 
à la masculinité ${ }^{2}$. Comme le soulignent de nombreuses études ${ }^{3}$, les filles et les garçons, dès leur naissance, font face à des interactions et des situations qui les font se développer différemment, tant au niveau corporel qu'au niveau mental, selon leur appartenance de sexe. Ainsi, ils ne sont pas soumis à la même éducation concernant la prise d'espace spatial et sonore, l'affirmation de soi ou encore l'expression des sentiments - et notamment l'agressivité. Cette socialisation différenciée, à laquelle les enfants participent activement, passe par une distribution spécifique des espaces et des matérialités ${ }^{4}$. Par exemple, la répartition des jouets (poupée versus arme en plastique) conduit à développer un certain rapport au monde puisque ces objets suscitent des interactions et des prises d'espace différentes voire opposées. Cela contribue à asseoir un ordre genré des attitudes corporelles et mentales et à maintenir une "naturalisation " des compétences dites féminines et masculines comme l'attention aux autres ${ }^{5}$ pour les premières et la prise de risque pour les seconds $s^{6}$. Le tir est intéressant à interroger sous l'angle du genre puisqu'il permet la manipulation d'objets, les armes, auxquelles les femmes n’ont généralement pas accès ${ }^{7}$.

De plus, le tir sportif s'inscrit dans un environnement - le sport - qui a été historiquement et socialement construit comme masculin ${ }^{8}$, prônant un

2. Michael Messner et Donald SABo, Sport, men, and the gender order: critical feminist perspectives, Champaign, Human Kinetics Books, 1990 ; George Mosse, The Image of Man. The Creation of Modern Masculinity, New York/Oxford, Oxford University Press, 1996.

3. Elena Belotti, Du côté des petites filles, Paris, Édition des femmes, 1974. Barrie Thorne, Gender play, New Brunswick, 1993 ; Anne Dafflon-Novelle (dir.), Filles-garçons : socialisation différenciée ?, Grenoble, Presses universitaires de Grenoble, 2006.

4. Nancy Henley, Body Politics: Power, Sex, and Nonverbal Communication, Upper Saddle River, Prentice Hall, 1977 ; Geneviève Cresson, «Indicible mais omniprésent : le genre dans les lieux d'accueil de la petite enfance ", Les Cahiers du genre, n²4, 2010, p. 15-33 ; Mona Zegaï, « La mise en scène de la différence des sexes dans les jouets et leurs espaces de commercialisation ", Les Cahiers du genre, $n^{\circ}$ 49, 2010, p. 35-54; Mona ZEGAï, « Du jeu dans les catégories de genre ? Le jouet comme outil de socialisation sexuée et de définition du champ des possibles au centre des interactions familiales ", in Sabrina Sinigaglia-Amadio (dir.), Enfance et genre. De la construction sociale des rapports de genre et ses conséquences, Nancy, Presses universitaires de Nancy, 2014.

5. Colette Guillaumin, Sexe, race et pouvoir, Paris, Côté-femmes, 1992.

6. Nicolas Penin, Les sports à risque. Sociologie du risque, de l'engagement et du genre, Arras, Artois Presses Université, 2012.

7. Paola TABET, «Les mains, les outils, les armes », L’Homme, n 19, 1979, p. 5-61 ; Jenifer CARLson, "Carrying Guns, Contesting Gender", Contexts, n 14, 2015, p. 20-25.

8. Vrada Burstyn, The Rites of Men: Manhood, Politics, and the Culture of Sport, Toronto, University of Toronto Press, 1999. 
modèle viril de la performance et de la réussite ${ }^{9}$. Dès la fin des années 1970, dans les pays anglo-saxons mais également dans les milieux francophones et germanophones ${ }^{10}$, de plus en plus de travaux en sociologie du sport se sont intéressés aux trajectoires des femmes et à leur difficulté d'accéder à de nombreux sports ${ }^{11}$, puis aux obstacles à atteindre les hauts niveaux de compétition ou la professionnalisation ${ }^{12}$. Ils ont également montré comment l'intégration progressive - en dents de scie - des femmes dans différents sports s'est accompagnée de la mise en place de règles et de conditions différentes de celles des hommes, de façon à ne pas pouvoir être comparées à ces derniers ${ }^{13}$. Cela a permis et permet encore de maintenir des croyances associées aux corps des femmes et des hommes en rendant visibles des différences corporelles ${ }^{14}$. Les filles et les garçons ne pratiquent donc généralement pas les mêmes activités physiques et n’y sont pas encouragés de la même manière ${ }^{15}$. Le tir ne fait ici pas exception. À cet égard, l'histoire du tir, et plus précisément du skeet ${ }^{16}$, aux Jeux Olympiques est intéressante. Introduite en 1968, la compétition devient mixte de 1972 jusqu'en 1992, année où une tireuse chinoise, Zhang Shan, bat le record du monde. Dès l'édition suivante, la compétition est à nouveau réservée uniquement aux hommes et les femmes peuvent concourir entre elles dès 2000. Ainsi, le fait qu'une femme batte des concurrents hommes amène à la redéfinition des conditions de participation et de pratique en mixité. En 2016, selon la

9. Il a en effet été démontré que le sport, dès lors qu'il s'est organisé et diffusé, a été un vecteur d'exclusion, car s'adressant à un nombre restreint d'individus pensés comme capables (physiquement, mentalement et moralement) : des hommes, jeunes et blancs issus des public schools, dénotant l'appartenance à une classe aisée et " urbanisée ». Voir notamment les travaux de Patrick Clastres et Paul Dietschy, Sport, culture et société en France du XIX' siècle à nos jours, Paris, Hachette, 2006 ; Sébastien Darbon, Diffusion des sports et impérialisme anglo-saxon, Paris, MSH, 2008.

10. Susan BANDy, "Gender and sports studies: an historical perspective", Movement \& Sport Sciences, $\mathrm{n}^{\circ} 86,2014$, p. $15-27$.

11. Suzanne LABERGE, "Les rapports sociaux de sexe dans le domaine du sport : perspectives féministes marquantes des trois dernières décennies ", Recherches féministes, $n^{\circ} 17,2004$, p. 9-38.

12. Christine Mennesson, "Le gouvernement des corps des footballeuses et boxeuses de haut niveau ", Clio, n 23, 2006, p. 179-196.

13. Annick Davisse et Catherine Louveau, Sports, école et société : la différence des sexes, féminin, masculin et activités physiques, Paris, L'Harmattan, 1998.

14. Raewyn Connell, Masculinities, Berkeley/Los Angeles, University of California Press, 1993.

15. Voir par exemple Julien Bois et Philippe Sarrazin, "Les chiens font-ils des chats? Une revue de littérature sur le rôle des parents dans la socialisation de leur enfant pour le sport ", Science et Motricité, n 57, 2006, p. 9-54.

16. Le skeet est une épreuve olympique où sont lancés des plateaux d'argile dans une surface définie, sur lesquels le ou la concurrente doit tirer à l'aide d'un fusil. 
fédération suisse de tir, les femmes sont $13 \%$ à être licenciées au tir avec armes à feu ${ }^{17}$. Elles sont environ $40 \%$ de licenciées au tir à l'arc mais il y a de grandes variabilités selon la discipline et les techniques apprises comme nous le verrons.

Le tir est donc ambivalent : il ouvre à de nouvelles postures et techniques corporelles pour les femmes - et certains hommes -, leur donne en partie accès à des objets et des qualités plutôt associés à la masculinité mais reste tout de même un espace largement investi par les hommes, se basant sur une tradition du tir qui se faisait entre hommes et ayant de fortes connexions avec le domaine militaire en Suisse. L'enjeu ici sera de montrer comment le tir à l'arc et le tir avec armes à feu travaillent la subjectivation des individus et permet de penser la formation de subjectivités spécifiques. En premier lieu, je vais aborder la manière dont a été théorisée l'articulation entre genre, objets et constitution de la subjectivité afin de dégager quelques pistes conceptuelles. En second lieu, je m'interrogerai sur l'existence ou non d'une division sexuelle des outils et des techniques sur mon terrain du tir sportif ${ }^{18}$. Quelles armes et techniques pour quels corps ? L'étude des relations entre matérialités, techniques et genre me permettra ensuite de montrer comment les matières contenues dans les objets (tels que le bois et le métal par exemple) jouent un rôle dans l'acquisition de techniques particulières et peuvent renforcer ou désamorcer des effets de genre. Cet article contribue donc à mettre en lumière des techniques associées à l'apprentissage et à la pratique du tir sportif qui peuvent être marquées par le genre, dans le sens où elles sont associées à la féminité ou à la masculinité, selon les situations, les personnes en présence et les matérialités en jeu.

17. Il est difficile d'avoir des chiffres pour la pratique de loisir puisqu'il n'existe pas de statistiques centralisées en Suisse et que c'est une pratique très populaire qui se fait aussi hors des fédérations sportives (surtout le tir avec armes à feu). Cela justifie d'autant plus d'observer les lieux de pratique pour quantifier la présence effective des femmes et leur intégration.

18. Danielle Chabaud-Rychter et Delphine Gardey, L'engendrement des choses : des hommes, des femmes et des techniques, Paris, Archives contemporaines, 2002. 


\section{Genre et « sujet-avec-ses-objets "}

La recherche autour de la culture matérielle et le genre connaît un relatif succès qui est récent ${ }^{19}$. Pourtant, Mauss évoquait déjà dans ses observations le fait que :

SC l'homme serre normalement le poing le pouce dehors, la femme le serre le pouce en dedans ; peut-être parce qu'elle n'y a pas été éduquée, mais je suis certain que, si on l'éduquait, ce serait difficile. Le coup de poing, le lancer du coup sont mous. Et tout le monde sait que le lancer de la femme, le jet de pierre est, non seulement mou, mais toujours différent de celui de l'homme : plan vertical au lieu d'horizontal. ${ }^{20}$

Cette différenciation dans les corps et dans la manière d'appréhender voire de manipuler la matérialité alentour s'expliquerait selon Mauss par le fait que les enfants ne reçoivent pas le même "entraînement " partout ${ }^{21}$, et que cet entraînement est différent selon le sexe.

Pour aller plus loin, Marie-Pierre Julien et Céline Rosselin ${ }^{22}$ parlent d'un "sujet-et-ses-objets ", mettant en avant l'importance de penser le sujet comme ne pouvant être dissocié de la matérialité environnante. Cette formulation se focalise sur l'implication de la matérialité dans la formation des subjectivités des individus. Dans cet article, je m’intéresse principalement aux objets du tir : les armes. Les objets ne doivent plus être considérés comme des « échantillons ${ }^{23}$ " ou n'être qu'utilisés pour démontrer quelque chose plutôt que d'être étudiés pour eux-mêmes ${ }^{24}$, ils font partie de nos

19. Anne Montjaret, "Objets du genre et genre des objets en ethnologie et sociologie françaises", Clio, n' 40, 2014, p. 153-170. Notre article s'intéresse au genre tout en n'oubliant pas que d'autres dynamiques sociales telles que la classe sociale, l'âge, l'ethnie peuvent entrer en jeu.

20. Marcel Mauss, "Les techniques du corps ", communication présentée lors de la Société de Psychologie française, Paris, 1934 puis publiée dans Journal de Psychologie, n 32, 1936, p. 11.

21. Pour creuser la transmission du geste technique et l'influence du contexte d'apprentissage, voir l'ouvrage collectif de Blandine BRIL et Valentine Roux, Le geste technique : Réflexions méthodologiques et anthropologiques, Toulouse, Erès, 2002. La question du genre n'est cependant pas traitée dans ces travaux.

22. Marie-Pierre Julien et Céline Rosselin (dir.), Le sujet contre les objets... tout contre : Ethnographie de cultures matérielles, Paris, CTHS, 2009.

23. Ibid.

24. Sara Ahmed, Queer Phenomenology: Orientations, Objets, Others, Durham/London, Duke University Press, 2006. 
expériences corporelles. Certaines recherches ${ }^{25}$, notamment anthropologiques, ont montré comment les objets peuvent contribuer au quotidien à créer des sujets genrés en postulant que les objets sont déjà eux-mêmes genrés car " porteurs de connotations de féminité et produisant différentes techniques corporelles aptes à leur manipulation ${ }^{26}$ ". Cependant, la plupart de ces analyses se sont surtout intéressées à l'aspect symbolique des objets, qui expliquerait une division sexuelle de ceux-ci et moins à ce que cela fait aux corps et aux subjectivités de celles et ceux qui les manipulent.

D'autres chercheuses, se positionnant dans le champ des sciences et des techniques, se sont penchées sur "la co-construction du genre et des technologies ainsi qu'aux dynamiques d'exclusion dans lesquelles sont imbriqués les corps et les technologies ${ }^{27}$ ". Ainsi, parce qu' elles possèdent certaines prescriptions, les technologies amèneraient à des discriminations qui excluent symboliquement et/ou matériellement des individus : un escalier pour une personne en fauteuil roulant par exemple. Ici la technologie est conçue comme "appartenant pleinement à son corps : on doit pouvoir s'identifier (visuellement, tactilement et affectivement) avec son corps technologiquement transformé $»^{28}$. Il y aurait donc un travail d'incarnation, c'est-à-dire « le fait que [la technologie] soit vécue de manière transparente aux niveaux sensoriel et cinétique ${ }^{29}$ et un travail d'incorporation, relatif à des normes sociales. Si cette approche a le mérite de montrer comment certains objets peuvent exclure des corps, elle postule que dès lors que les corps semblent physiquement aptes à la manipulation, il y a une incarnation qui se fait automatiquement. Mais l'incarnation est-elle exempte d'apprentissages liés à des normes sociales ? Les femmes possèdent-elles un même éventail de sensations et d'émotions que les hommes, face à certains objets, et plus particulièrement en contact des armes ?

25. Voir par exemple Marianne Gullestad, "Home decoration as popular culture. Constructing homes, genders and classes in Norway", in Teresa Del Valle (dir.), Gendered Anthropology, Londres, Routledge, 1993, p. 128-161 ; Elisabeth Anstett, Marie-Luce GÉLARD, Les objets ont-ils un genre? Culture matérielle et production sociales des identités sexuées, Paris, Armand Colin, 2012.

26. M.-P. Julien et C. Rosselin (dir.), Le sujet contre les objets, op. cit., p. 63.

27. Lucie Dalibert, "Technologie ", in Juliette Rennes, Encyclopédie critique du genre, Paris, La Découverte, 2016, p. 628.

28. Ibid., p. 636.

29. Ibid. 
La phénoménologie féministe me semble être une piste intéressante pour

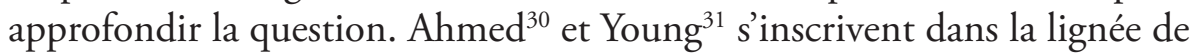
Merleau-Ponty pour qui la formation du sujet passe par une façon d' "e êtreau-monde » corporellement, qui est tournée vers l'extérieur et donc à la matérialité environnante. Notre relation au monde serait de l'ordre de la perception, de la sensation et ce lien serait "stable et continu " ${ }^{32}$. C'est sur ce point-là notamment qu'Ahmed et Young s'éloignent de MerleauPonty car elles expliquent que les femmes apprennent à avoir un rapport au monde spécifique, elles sont orientées d'une certaine manière et cela ne peut être stable puisque dépendant des situations. De plus, cela est travaillé par des normes de genre, qui déterminent en partie comment les hommes et les femmes se meuvent dans un tel espace car ceux-ci n'ont pas les mêmes accès, qui sont socialement définis. Ainsi, il existe des objets hors de portée, tandis que d'autres sont à proximité, voire "sous la main ». Certains ne demandent qu'une infime préparation à leur manipulation alors que d'autres sont plus techniques, mais selon la manière dont ils sont associés à la féminité, ils sont rendus ou non accessibles aux femmes (par exemple les appareils électroménagers versus les outils de menuiserie). En effet, les matérialités demandent des connaissances corporelles spécifiques et du moment que ces corps ne sont pas traités socialement de la même manière car étant pris dans des représentations genrées, ils développent un rapport différent aux objets. Si effectivement certains objets sont attribués aux hommes et aux femmes, ils ne seraient pas en soi genrés mais participeraient plutôt à une "spatialité du genre " ${ }^{33}$, mettant en exergue le fait que le genre ne produit pas les mêmes effets selon les situations.

Afin d'être plus explicite, je prends l'exemple du jeter de balle, explicité par Young ${ }^{34}$. L'auteure montre comment les filles ont appris à utiliser leur corps de manière différente et comment cela a développé un rapport à soi spécifique. En effet, lorsqu'elles jettent une balle, elles ont tendance à utiliser seulement une partie de l'avant de leur bras, là où les garçons utilisent l'entièreté du membre et le haut de leur corps. Cette différenciation

30. S. Ahmed, Queer Phenomenology, op. cit.

31. Iris Marion Young, "Throwing like a girl: A phenomenology of feminine body comportment motility and spatiality", Human Studies, n 3, 1980, p. 137-156.

32. Maurice Merleau-Ponty, Phénoménologie de la perception, Paris, La librairie Gallimard, 1945.

33. S. Ahmed, Queer Phenomenology, op. cit.

34. I. M. Young, "Throwing like a girl”, op. cit. 
dans le geste se répercute dans la propulsion de la balle et donc du résultat de la performance corporelle, jugée plus ou moins puissante et efficace. Cette analyse a le mérite de montrer que les apprentissages de la préhension et de la manipulation des objets participent à maintenir un ordre de genre et à valider une différenciation des corps, et donc des résultats, des femmes et des hommes. Des travaux ont montré que la balle est connotée comme masculine surtout lorsque celle-ci est lourde et projetée, puisqu'associée à la force ${ }^{35}$. Cependant, la projection d'objet n'est pas l'apanage des activités considérées comme masculines, en témoigne le twirling bâton. Néanmoins, lorsque la projection est liée à une question de force et de performance, elle a tendance à être plus facilement associée à la masculinité (lancer du marteau par exemple). Qu'en est-il donc des armes, objets associés à la virilité ? Est-ce que les hommes et les femmes manipulent les mêmes objets dans le cadre du tir sportif et en quoi le contexte joue-t-il un rôle dans cette « orientation " ?

\section{Une division sexuelle des armes?}

Selon Paola Tabet ${ }^{36}$, il existerait une division sexuelle des outils et des techniques dans nos sociétés occidentales et les armes ainsi que leur manipulation seraient assignées aux hommes. Pour justifier cette observation, l'anthropologue prend l'exemple des premières formes de sociétés et déconstruit l'idée selon laquelle les hommes chassaient tandis que les femmes cueillaient. Elle explique que les femmes aussi participaient à la chasse mais elles n'avaient pas accès aux outils pour tuer l'animal. Ainsi, elles apprenaient à le piéger et à l'assommer. Elles usaient donc de techniques associées aux objets qu'elles avaient en leur possession (gourdins, cordes, par exemple) et validaient ainsi une division des tâches et des rôles des femmes et des hommes dans la société.

Le lien entre virilité et armes est encore soutenu institutionnellement en Suisse, puisque le service militaire est obligatoire pour tout citoyen homme à sa majorité. Certes, pas toutes les recrues se retrouvent dans des sections où il y a manipulation d'armes, mais il s'agit d'une minorité. En outre,

35. Annick Davisse et Catherine Louveau, Sports, école et société : la différence des sexes, féminin, masculin et activités physiques, Paris, L'Harmattan, 1998.

36. P. TABET, «Les mains, les outils, les armes ", op. cit. 
un grand nombre de productions culturelles mettent en scène plus facilement un homme dans la peau d'un individu qui porte des armes qu'une femme ${ }^{37}$. Mais cela doit être nuancé puisque des figures de femmes en armes apparaissent de plus en plus même si la plupart du temps, elles n'ont pas un contrôle sur l'arme mais les utilise pour se défendre ${ }^{38}$, allant alors dans le sens d'une partielle inadéquation entre femmes et armes. Alors même que l'arme est désinvestie dans le tir sportif de ses buts premiers qui sont de se défendre ou de tuer, cela amène-t-il pour autant à une ouverture à la manipulation de toutes sortes d'armes pour les femmes ${ }^{39}$ ?

Dans le tir à l'arc, trois types d'arc sont principalement utilisés : le longbow, le recurve et l'arc à poulie. Le longbow est un arc dit nu, c'est une branche en bois en une seule partie et sur laquelle il n'y a pas possibilité d'ajouter des accessoires. Une corde est placée de bout en bout et contrairement aux autres types d'arc, il n'y a pas d'indication sur la corde du placement de la flèche et il faut tenir la flèche avec sa main lorsque l'on bande l'arc. Le longbow est facilement associé à des figures tel que Robin des Bois, à la chasse et à " la manière la plus naturelle de faire du tir à l'arc" (Natacha, 45 ans). Le recurve est un arc qui peut être " accessoirisé " jusqu'à devenir un arc olympique (avec stabilisateur et viseur). Cet arc se présente en trois parties - deux branches et une poignée - qu'il faut monter à chaque entraînement. Les archer·ère-s peuvent donc adapter l'arme à leur morphologie en choisissant une certaine puissance des branches et un type de poignée. De plus, au fur et à mesure de l'apprentissage du tir, il est possible de changer les branches pour que l'arc gagne/perde en puissance. Enfin, cet arc possède un repose-flèche, c'est-à-dire que le ou la pratiquante peut poser la flèche et ne pas se préoccuper de sa tenue sur l'arc lorsqu'il tire. Les doigts restent en effet seulement sur la corde. Quant à l'arc à poulie, il se présente sous une forme plus compacte et comporte deux poulies en haut et en bas. Le geste pour tirer ressemble davantage à celui du tir au pistolet puisqu'il s'agit de tenir une sorte de gâchette que l'on a aux doigts, qu'on accroche à la corde et qu'on actionne au moment du tir. Les doigts ne sont

37. Jean-Jacques Courtine (dir.), Histoire de la virilité, t. 3, Paris, Seuil, 2015.

38. Karen Ritzenhoff et Jakub Kazecki, Heroism and Gender in War Films, New York, Palgrave Macmillan, 2014.

39. Il existe une multitude de sortes d'armes dans les deux pratiques étudiées (le tir à l'arc et le tir avec armes à feu). Cette liste est faite à partir de mes observations et des armes avec lesquelles les personnes avec qui j'ai pratiqué étaient le plus souvent en contact. 
donc ni en contact avec la corde, ni avec la flèche. L'esthétique de l'arc à poulie est souvent identifiée à un outil technologique et puissant.

Ces différents arcs ne sont pas répartis de la même manière selon le sexe des pratiquant.e.s. On trouve la plus grande majorité d'hommes (plus de $90 \%$ ) avec l'arc à poulie, celui-ci ayant " un visuel hyper masculin, alors qu'en principe tu as besoin de moins de force pour une même puissance de tir " (Caroline, 38 ans). Le fait que le geste de tir soit comparé au tir au fusil justifie également pour la plupart des pratiquant.e.s le fait que les femmes s'y intéressent moins, partant de l'idée que la virilité et les armes à feu sont liées. Environ $20 \%$ de femmes font du longbow. L'association de cet arc à la chasse semble être un obstacle à l'intégration des femmes. Cependant, le fait qu'il existe des compétitions dans des forêts notamment (où les archer.ère.s tirent sur des animaux en mousse ou sur des cibles classiques) permet une présence des femmes plus grandes, celles-ci étant associées plus facilement à la nature ${ }^{40}$. Ainsi, une motivation commune est partagée par les deux sexes : l'appel de la nature. Celle-ci n'est pas remise en cause ou ne nécessite pas davantage d'explications puisque faisant appel à une " communauté imaginée ${ }^{41}$ ". Cependant, si une femme souhaite chasser, elle devra souvent se justifier, ce qui ne serait pas le cas pour un homme, comme le témoigne Marie, chasseuse de 62 ans que j'ai interviewée. Cela réaffirme ici les normes de la féminité qui associent les femmes à la douceur et à l'aversion de tuer ou de faire du mal, et a contrario la masculinité à la violence. Enfin, l'arc recurve est un arc qui permet une mixité entre hommes et femmes. Cela s'explique par le fait que «c'est l'élément qui s'adapte à la morphologie, tu choisis de faire ton arc selon ta puissance " (Tom, 45 ans). Le fait que l'arc soit adaptable neutralise donc en partie l'argument de la force et permet de créer une variabilité d'arcs autant qu'il y a d'archer.ère.s et ainsi d'accueillir une diversité de corps. De plus, n'étant pas associé à un domaine viril (militaire, technique, chasse), l'arc recurve est davantage pratiqué par les femmes. Le geste technique est également vu comme moins difficile (surtout au niveau de la puissance) alors que cela dépend des branches choisies. Enfin, sa manipulation est pensée comme très liée à une question d'apprentissage puisqu'il s'agit de mettre ses doigts sur la corde à un certain endroit pour tirer à une certaine

40. C. Guillaumin, Sexe, race et pouvoir, op. cit.

41. Eduardo Archetti, Masculinities. Football, Polo and the Tango in Argentina, Oxford/New York, Berg, 1999. 
distance de tir. Avec le longbow, la manipulation est articulée à la question de la force et à la technique du tir instinctif, supposant des qualités pensées comme naturelles - par conséquent il est davantage choisi par les hommes. Cependant, les hommes ont tendance à surestimer leur puissance et à prendre des arcs plus puissants que ce que leur corps est effectivement apte à tirer. Sachant que plus un arc est puissant, plus il est précis, cela a une influence sur les résultats. Ces observations vont dans le sens d'une division sexuelle des armes, selon les différentes représentations associées aux gestes et aux activités liées à celles-ci.

Dans le tir avec armes à feu, il est difficile de dégager uniquement quelques catégories, je vais donc rendre compte des principales armes utilisées dans le cadre du tir sportif dans les clubs et compétitions que j'ai suivis. Tout d'abord, il existe des armes de petits calibres et de gros calibres et la répartition genrée est assez claire : les femmes ont tendance à choisir des armes plus petites, plus légères, donc moins bruyantes et qui supposent une plus courte distance d'avec la cible. De plus, on dispose du tir à air comprimé qui se tire avec un certain type de balles, qui n'induit pas de recul (le fait que l'arme, sous la puissance du coup, revienne en arrière) et qui est davantage pratiqué par les femmes. Marie, 27 ans, explique que certains hommes « aiment bien tirer avec du gros calibre, car ça fait du bruit, c'est dangereux car ce sont des balles puissantes, parce que ça déménage et que tu as un recul fou et tu éjectes ». Le recul est très fort dans l'épaule par exemple avec le fusil d'assaut. Ainsi, on retrouve plus facilement des hommes avec des fusils et des armes gros calibres et des balles puissantes. Les sensations du doigt sur la détente sont aussi considérées comme plus intenses lorsque le recul est plus fort comme le constate Marie : "Y a des hommes qui disent qu'avec le tir à air comprimé (sans recul), ben ça fait simplement pouf après le tir, c'est pas intéressant, on ressent pas une puissance de l'arme ». Lors d'une initiation de tir, un moniteur me montre un pistolet $22 \mathrm{~mm}$, utilisé par la police suisse, qui est considérée comme puissante et me dit : "ce n'est pas une arme pour les femmes" (Michel, 45 ans).

Cependant, même si on peut dégager très clairement une division sexuelle des armes, elle est le fruit de " choix » et d'expériences vécues qui cadrent les décisions des pratiquant.e.s. Ces dernier.ère-s s'orientent et sont orienté.e.s vers certains objets ${ }^{42}$. Ainsi, Michel, alors qu'il m'indique que l'arme

42. S. Ahmed, Queer Phenomenology, op. cit. 
que j'ai devant moi n'est pas faite pour les femmes, me propose tout de même de l'essayer. Marie est à côté de moi et me dit qu' elle n'aime pas cette arme " car elle a toujours peur de se péter le nez avec le recul ». Je l'essaie et j'appréhende le fait que le contexte ne soit pas favorable à bien vivre l'expérience. Étant crispée, je serre trop l'arme avec ma main et je reçois tout le recul dans celle-ci, validant le fait que cette arme est puissante et peut blesser ; en quelque sorte qu'elle ne me convient pas, même s'il s'agit d'une première expérience de manipulation. Ici aussi une division sexuelle des armes est visible, qui se joue sur les gestes et les sensations, avec par exemple la peur des blessures - plutôt ressentie par les femmes - ou le bruit recherché ou non. Cela va dans le sens d'une différenciation quant à la prise d'espace sonore et la prise de risque selon le sexe. Le fait de côtoyer des objets considérés socialement comme dangereux et qui sont cadrés par des mesures de sécurité semble renforcer cette division, avec des armes considérées comme plus ou moins difficiles à manipuler pour les femmes car plus puissantes.

\section{Des frontières genrées perméables ?}

Cependant, il serait erroné de voir la division sexuelle des armes comme un processus automatique et transversal à toutes situations. En effet, celle-ci est très dépendante du contexte, des matérialités et des autres personnes en présence. Je vous propose ici quelques exemples. Il existe en Suisse des cours intitulés "Jeunes tireurs " qui s'adressent aux jeunes de 15 ans à 20 ans (l'âge peut varier selon les régions ; les cours se passent hors du cadre scolaire). Ces entraînements sont mixtes et seul le fusil d'assaut est utilisé. Le choix de cette arme s'explique par le fait que l'armée les fournit de manière gratuite aux sociétés de tir pour effectuer ces cours. Il s'agit du fusil d'assaut de l'armée suisse, que les recrues doivent apprendre à manier durant leur service militaire obligatoire. De manière intéressante, les femmes et les hommes tirent donc ensemble et participent à des concours en groupe. Cette arme contient un grand recul et tire sur des cibles à $300 \mathrm{~m}$. Plusieurs positions de tir sont possibles : debout, un genou à terre ou couché. Mais selon mes observations et les dires de mes enquêté.e.s, la majorité du tir avec le fusil d'assaut se fait couché. Comme me l'explique Nathalie (29 ans), qui a participé à ces cours étant plus jeune, "le fait d'être couchée permettait de dire qu'on était aussi capables les femmes. On 
pouvait avoir plus d'appuis pour moins reprendre le recul dans l'épaule ». Ainsi, selon le geste et la posture adoptés, une partie de l'argumentaire selon lequel le corps des femmes seraient moins aptes à tirer et à prendre le recul s'affaiblit. Le fait d'être dans un groupe de jeunes peut également jouer un rôle : un grand nombre de filles adolescentes font du sport, et notamment avec les garçons mais on constate une chute des effectifs dès la majorité atteinte et dès lors, une division des activités sportives beaucoup plus forte ${ }^{43}$. Dans le cas du tir, beaucoup de filles arrêtent après les cours de jeunes tireurs car elles ne sont pas appelées à tirer dans le cadre de l'armée et sont moins incitées que les garçons à continuer dans un sport où se manipulent des armes, surtout des armes à feu. Ainsi, il existe des espaces de pratique où les femmes sont intégrées au même titre que les hommes, et cela même s'il y a manipulation des armes de gros calibres.

Au sein du tir à l'arc, certaines armes comme le longbow, plutôt associé à la chasse comme on l'a vu, accueillent cependant un nombre croissant de femmes et notamment de femmes dès la trentaine d'années alors que la pratique est vue comme difficile, et elle l'est effectivement. Considérées comme plus élégantes dans leurs gestes, les femmes rendraient le tir " plus beau " (Théo, 52 ans). Cependant, on retrouve une césure entre femmes et hommes, les premières étant plutôt pensées comme capables du geste élégant alors que chez les hommes il s'agirait davantage d'une aptitude au geste technique. On comprend donc que cela peut défavoriser l'apprentissage à des fins compétitives pour les femmes. Une autre représentation liée à des normes genrées a aussi cours dans de nombreuses interactions et paroles de mes enquêté.e.s, notamment dans le tir avec armes à feu, celui-ci suggère que les femmes seraient naturellement plus précises que les hommes. Ainsi, la plupart des moniteurs rencontrés soulignent l'importance d'avoir des femmes dans leur rang. On voit ici que les représentations sociales associées à la féminité ne fonctionnent pas d'office comme un obstacle à la pratique et à la manipulation de certaines armes. Plus encore, certains sports comme le golf ont vu les femmes avoir de meilleurs scores lors de gestes qui devaient être davantage précis que puissants, démontrant que l'acquisition de techniques spécifiques liées à des normes genrées peut

43. Isabelle Courcy, Suzanne Labverge, Carine Erard et Catherine Louveau, « Le sport comme espace de construction sociale de la féminité : jugements d'adolescentes et d'adolescents concernant les filles qui pratiquent des sports de tradition masculine ", Recherches féministes, n 19, 2006, p. 29-61. 
amener les femmes à acquérir des bons résultats mais uniquement dans certains gestes, qui ne sont pas forcément jugés comme les plus importants (par exemple, la puissance du corps est globalement plus recherchée que la précision du geste) si l'on considère l'ensemble des pratiques sportives existantes.

L'influence des moniteurs et monitrices est également importante dans le "choix » des armes par les enquêté.e.s. Dans la plupart des initiations que j'ai pu faire dans le tir avec armes à feu, toutes sortes d'armes m'étaient proposées. Cela peut être dû aussi à mon statut de personne faisant une recherche sur le tir mais j'ai pu remarquer que de manière générale, les initiations pour les femmes et les hommes se ressemblaient quant à l'éventail d'armes proposées à l'essai. Il faut tout de même nuancer : si les objets sont effectivement mis à disposition, le discours qui encadre la manipulation peut jouer un rôle d'(in-)accès à certaines armes. L'exemple que j'ai donné précédemment dans lequel j'ai eu une mauvaise expérience avec une arme était peut-être dû au fait que je ne me sentais pas légitime à utiliser une telle arme puisque le moniteur m'avait dit juste avant de tirer qu'elle n'était pas «faite pour les femmes ». De plus, je n'avais jamais été en contact avec des armes, j'avais donc une plus grande attente et attention par rapport à l'encadrement. Enfin, les autres personnes en présence peuvent également jouer un rôle en étant plus ou moins encourageantes.

Au final, la situation amène les personnes à se sentir plus en moins invitées et encouragées à manipuler certaines armes, dénotant la force des normes genrées sur les corps des femmes et des hommes et sur la manière dont elles et ils se perçoivent. Par exemple, un moniteur m’a expliqué que même s'il donne les mêmes indications aux femmes et aux hommes lors des initiations de tir, les hommes ont tendance à moins attendre la fin des explications et à tirer davantage " en rafales ». Cela irait dans le sens d'une meilleure aisance avec une arme, sans que cela se vérifie pour autant dans les résultats lors des initiations. De plus, comme nous l'avons vu, les armes puissantes sont souvent vues comme en inadéquation avec les corps des femmes, alors plutôt perçus comme fragiles. Pourtant, pour les personnes plus âgées qui ne peuvent plus tirer avec des armes à feu sans aide, des solutions sont mises en place comme l'existence d'une barre qui permet de maintenir le bras droit pour tirer. On peut donc poser l'hypothèse que la force des représentations genrées sur la faiblesse des corps exerce une influence sur les femmes et les empêche parfois de tirer avec certaines 
armes alors que lorsque la faiblesse des corps est avérée et concerne des hommes tireurs, ceux-ci sont encouragés à continuer à tirer avec des aides. Le fait de " faire corps » avec son arme ne relève donc pas seulement d'une expérience vécue corporelle seul.e mais met en jeu autrui, la matérialité et des représentations sociales associées à celle-ci. Enfin, " choisir " son arme peut également passer par les matières qui composent les armes et qui, elles aussi, peuvent être liées à des normes de genre.

\section{Entre le bois et le métal : les matières qui font les tireurs/euses}

Les différentes matières peuvent déterminer le choix d'une arme et jouer sur la manière dont l'expérience va être ressentie. Un enquêté, qui fait du tir à l'arc et du tir au pistolet m'explique :

Alors moi la différence que je vois entre le pistolet et l'arc c'est que le pistolet, bon il peut être fait en plastique, en bois ou en métal mais ça reste quand même beaucoup du métal. Alors que l'arc c'est plus du bois, je sais pas si c'est le côté plus proche de la nature, qui peut être gravé... c'est ça le côté arc qui peut être joli quoi. Bon c'est sûr dans les films, ils sont tous gravés, magnifiques et tout. Alors que le pistolet... ouais c'est vraiment aussi le métal qui fait aussi que ça fait froid. Cette distance, le froid alors que le bois, ça fait plus le chaud.

Pour lui, le métal crée une distance que l'on ne retrouve pas avec le bois. Valérie, archère en longbow va plus loin dans son rapport à l'arc et à la matière :

Ça m'est arrivé qu'une fois, mais c'est une sensation que j'aimerais bien revivre une fois. On a tous vécu ce moment où on a armé, on n'a pas lâché, mais on sait que la flèche sera bonne. Et elle est bonne! Comme quand on sait qu'elle sera à côté, et elle est à côté. Et là, non seulement je savais qu'elle était bonne, mais quand je l'ai lâchée, j'ai eu l'impression d'être la flèche, c'est comme si je partais avec la flèche. Mais c'était une sensation que j'ai vécue qu'une fois, comme si j'étais devenue la flèche! J'ai jamais revécu ça. 
Ici il ne s'agit pas seulement d'un prolongement du corps qui serait facilité par des matières associées à la nature telle que le bois, mais d'une projection dans la matière même. Cette fascination pour la projection de la flèche, pour cette association avec la nature est apparue à plusieurs reprises dans mon terrain de tir à l'arc, que ce soit de la part des femmes et des hommes ${ }^{44}$. A contrario, aucun.e tireur/euse ne m'a parlé d'un "devenir balle ", posant l'hypothèse que l'identification avec la matière serait plus aisée avec du bois qu'avec du métal, celles-ci étant soumises à des représentations différentes et ouvrant un éventail de sensations aux deux sexes. Ici le genre ne semble pas jouer un rôle saillant dans le vécu du rapport avec le bois, il s'agit davantage d'un certain groupe de personnes qui considère avant tout le tir comme une philosophie. Pour elles, c'est une manière de se mesurer à soi (et non aux autres) et elles vont donc plus facilement choisir une technique de tir à l'arc qui a le moins d'accessoires possible et «la plus instinctive possible». L'aspect d'introspection n'est pas un obstacle pour la pratique des femmes, au contraire, puisque relié à un trait de la féminité qui serait le fait de pouvoir s'écouter et exprimer ses émotions ${ }^{45}$. Cependant, dès lors que l'aspect compétitif reprend le dessus, on retrouve moins de femmes, moins socialisées à ce rapport au monde.

Le métal ne suscite pas forcément une distance mais est souvent associé à une certaine part d'incontrôlable que j'ai peu retrouvée avec l'arc. Certains tireurs y vouent tout de même un certain attachement, surtout les collectionneurs ou ceux qui ont une certaine connexion avec le domaine militaire. Certains verront donc une certaine corvée à devoir nettoyer l'arme à chaque session de tir alors que pour d'autres, c'est un plaisir de la démonter : «Pour moi une arme à feu, c'est avant tout une belle mécanique en métal. Je pourrais faire un parallèle avec l'horlogerie. »(Simon, 48 ans).

Enfin, la relation avec la matière varie selon deux visées principales : l'esthétique et la volonté de performer au niveau sportif. Ainsi, m’explique un facteur d'arc, les femmes vont choisir davantage des arcs en bois plus clair que les hommes. De plus, un marché commence à se développer d'armes à feu plus colorées, notamment avec des poignées roses. On voit donc que des stratégies marketing jouant sur les représentations sociales

44. J'ai pu observer ce phénomène surtout chez les tireurs/euses de tir instinctif et lors de mes observations dans un club de Kyûdô.

45. N. Henley, Body Politics, op. cit. 
genrées tentent de rendre les armes plus accessibles aux femmes. Quant à la dimension sportive, certains matériaux sont considérés comme plus performants. Par exemple, certains types de bois sont plus ou moins rigides. Le matériau des flèches et le choix entre carbone ou bois est également au cour de discussions, tout comme la longueur des plumes au bout de la flèche. La relation aux matériaux passe donc par un rapport spécifique à la pratique (plus ou moins investie sportivement ou non) et à une certaine relation affective à son arme. Pour beaucoup d'hommes, il s'agit d'un objet de sport mais avec lequel ils ont un lien qui dépasse la seule performance sportive (arc et armes à feu) alors que pour les femmes cela dépend si l'on parle d'armes à feu ou d'arcs. Les femmes auront plus de difficulté à voir l'arme à feu comme un objet avec lequel elles peuvent entretenir un rapport affectif. D’ailleurs, mon terrain est assez révélateur sur ce point : si la plupart des hommes ont acheté leurs armes à feu très vite après avoir commencé la pratique, les femmes ont rarement acheté par elles-mêmes leurs armes. Soit les armes avec lesquelles elles tirent sont un prêt, soit elles leur ont été offertes/conseillées par leurs conjoints ou moniteurs.

Comme on l'a vu, le tir implique un engagement corporel spécifique, des objets et des discours associés à ceux-ci pouvant cristalliser des enjeux de genre. La lecture en termes de genre et à partir de la manipulation de la matérialité a permis de montrer que si, effectivement, une division sexuelle des armes se révèle, elle ne peut être considérée comme transversale à toutes situations. L'influence des autres personnes en présence des moniteur-rice-s de tir, du matériau de l'arme et des différentes représentations sociales autour du corps des femmes et des qualités considérés comme féminines ne se joue pas de la même manière et avec la même intensité selon les contextes. L'apprentissage d'une certaine posture corporelle et la manipulation des armes ouvrent certes un espace où les représentations genrées peuvent être travaillées, davantage au niveau corporel que discursif. En effet, si le tir " permet de faire des choses féministes, d'essayer de tenir son corps de manière différente de que ce qu'on apprit socialement, autant en tant que femme qu'en tant qu'homme » (Caroline, 38 ans), la plupart des discours encadrant la pratique repose sur des croyances différenciées de ce qu'un corps d'homme et ce qu'un corps de femme est capable de faire, et donc de manipuler. 
Solène Froidevaux

\section{L'autrice}

Solène Froidevaux est doctorante en sociologie à l'Université de Lausanne, au Centre en Études Genre. Dans le cadre de sa thèse, elle analyse les processus de subjectivation des tireurs et tireuses sportives (tir à l'arc et tir avec armes à feu) en se focalisant sur la manière dont l'expérience corporelle du tir est articulée aux matérialités inhérentes à la pratique (en particulier les armes) et comment celle-ci est plus ou moins marquée par le genre selon les contextes. Contact : solene.froidevaux@unil.ch 